\title{
Towards Engineering Design Courseware
}

\author{
Robert W. Brennan, Marjan Eggermont, Mahnaz Shams \\ Department of Mechanical and Manufacturing Engineering \\ University of Calgary, 2500 University Dr. N.W., Calgary, AB, T2N 1N4 \\ Phone: +1-403-220-5798 \\ Email: rbrennan@ucalgary.ca
}

\begin{abstract}
In this paper we focus on how engineering design education material can be converted to engineering design courseware or CDEN web-based modules. More specifically, the paper addresses the characteristics and basic standards for modules, the module's objectives relative to engineering design competency, and key issues to be considered when developing modules.
\end{abstract}

\section{Introduction}

The general goals of the CDEN/RCCI network are to "enable the communication of best design practices between schools, promote the production and sharing of courseware, help inject more real design experiences into the university, and allow all schools to access the best available expertise in areas of detailed interest" [1]. In order to achieve these goals, it has been proposed that an e-learning approach is adopted that involves the joint development of webbased design courseware modules that can be shared by members of the CDEN community. This approach will require a merging of engineering pedagogy and information technology as the term "e-learning" implies [2]:

- “how is e-learning delivered?": via computer, using words (spoken or text) and pictures (still, video),

- "what does e-learning include?": both content and instructional methods that help students learn the content,

- "why use e-learning?": to help students reach personal learning objectives.

In the context of CDEN, the internet will be used to deliver design-directed learning [3] modules to improve engineering design competency in future graduates [4]. Although it is expected that CDEN module developers are experts in the content of their respective modules, their degree of expertise in the area of e-learning and, in particular, web-based teaching of open-ended design will vary considerably. Despite this, the success of the CDEN network ultimately rests on the quality, usability, and accessibility of these modules.

In this paper, we address this concern by focusing on how engineering design education material can be converted to engineering design courseware or CDEN web-based modules. More specifically, the paper will address the characteristics and basic standards for modules, the module's objectives relative to engineering design competency, and key issues to be considered when developing modules.

CDEN modules should contain certain basic information as a standard. For example, the module developer should state any prerequisite competencies required for the module, the module's relationship to the CDEN Tiers [3], and also provide appropriate metadata to enable searching and indexing (e.g., [5]). Modules should also be of an appropriate level of granularity (i.e., the size of the module) to enable online learning.

When deciding the best approach for web-based module design (e.g., web-based tools used, interactive methods, ...), it is important to consider the teaching objectives for the module. For the CDEN community, teaching objectives will be closely tied to engineering design competencies [4]. The notion of design competencies will also be useful for metadata design in order to allow users of the CDEN website to search and retrieve modules on this basis.

Other key issues that should be considered included guidelines of minimum standards for modules and mechanisms for peer reviewing (e.g., an online feedback mechanism could be used), guidance on combining web-based learning and face-to-face learning, guidance on effectively combining 
knowledge (tier 1), case studies (tier 2) and openended problems (tier 3I), and procedures for benchmarking modules.

The paper begins with background on CDEN modules, tiers and sorts. Next, we look at how the general concepts of e-learning can be applied in this area and illustrate this with various examples of CDEN design modules. In section 4 we tie these areas together and offer some suggested guidelines on how CDEN modules can be presented in a web-based format. The paper concludes with our thoughts on the next steps in the process of fostering the development of shared courseware in the engineering design community.

\section{CDEN Modules, Tiers and Sorts}

A central aspect of the CDEN project is the joint development of web-based design courseware modules that can be shared by members of the CDEN community. These courseware modules were at first considered to include lectures, case studies and openended design projects. The basic idea was to support existing faculty with design materials and encourage schools to collectively build the critical mass within a team environment needed to promote the importance of design and effect significant improvement in existing programs [1].

In order to concisely define the content of the CDEN modules a classification by "tiers" and "sorts" was defined as illustrated in Figure 1.

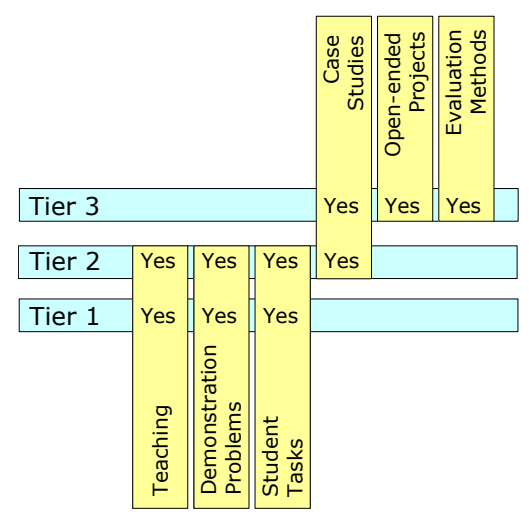

Figure 1. Matrix of Tiers and Sorts (adapted from [1])

The "tiers" classification is intended to organize CDEN modules over a spectrum from fundamental engineering science principles to open-ended engineering design exercises [1]:
- Tier 1: Modules at this level address fundamental principles of two specific types (a) a basic qualitative or quantitative relation or law from the engineering sciences or mathematics (e.g., conservation of momentum, Kirchoff's laws), or (b) in design science, an empirical relation or statement about the technical aspects of product development that is likely to be true in most cases (e.g., standardization of parts).

- Tier 2: Modules at this level addresses different technologies, methods and engineering practices (e.g., dimensioning, material selection, etc.).

- Tier 3: This highest-level tier is concerned with the integration of tier 1 and tier 2 modules to build toward various design modules (e.g., a case study such as an automated saw).

Associated with each tier is a "sort", or way in which the courseware module may be used. In particular, the sorts shown in Figure 1 are defined as follows [1]:

- Teaching Module: representative of a lecture.

- Demonstration Problem: representative of a worked exercise or illustration.

- Student Task: similar to a demonstration problem, but without a detailed solution.

- Case Study: presentation of the design experience to emphasize the interrelationships of various principles, technologies, and methods.

- Open-ended Project: a problem to exercise the students' ability to integrate principles, practices, methods, and technologies.

- Evaluation Method: a technique used to measure the performance of students.

The original thinking behind the web-based teaching modules was fundamentally sound. In particular, web-based modules appear to be an effective way of disseminating course material, and the web-based model of publishing works well with a wide range of thematic material. For example, the presentation can be as simple or as complex as is needed to convey the message. As well, there is also the ability for an interactive learning experience, which may be desirable in many cases.

The question that arises is how can engineering design instructors most effectively transform their teaching materials to online courseware or "CDEN modules"? As well, given that the module developers will come from a wide selection of engineering schools and disciplines, how can we maintain consistent courseware without requiring the authors to spend an inordinate amount of time getting up to speed on the latest e-learning technologies? Before looking 
at this problem in section 4 we provide an overview of some of the more general research on e-Learning.

\section{3. e-Learning Approaches}

E-learning is the delivery of a learning, training or education program by electronic means. E-learning involves using a computer or electronic device (e.g., a mobile phone) to provide training, educational or learning material.

E-learning can involve a greater variety of equipment than online training or online education: for as the name implies, "online" involves using the Internet or an Intranet. Additionally, CD-ROM and DVD can be used to provide learning materials [6].

E-learning allows the sharing of resources, can be used in either an asynchronous or synchronous way, and is place independent.

However, a greater emphasis must be placed on the delivery of e-learning material. Usability and instruction play an equally important role. Instruction, in this case, refers to the message, and usability to the 'wrapping'. Instruction is the doing and usability is how the doing is facilitated and optimized. Usability in an e-learning context refers to diverse things such as the platform specifications; screen layout; the navigational system and structure; the aesthetic qualities of a platform; and the traits that promote user-friendliness.

E-learning as seen through the eyes of instructional learning models, falls under the category of active learning; constructing new knowledge based on prior knowledge; real-world observation with real problems in real situations; constructing interpretations of observations; collaborating and cooperating with peers; and learners acting as facilitators/coaches to each other. These are all aspects that e-learning naturally promotes if the usability and the instruction are a well-balanced entity as illustrated in Figure 2.

Given this general model of the e-learning instructional design process, one may ask at this point, how can this be applied in the context of the CDEN tiers and sorts described previously? In terms of usability, each tier should be consistent. In other words, each tier should first and foremost include similar or identical style guidelines (for headings, numbers, names, punctuation, and capitalization), identity, graphics guidelines, screen designs, and page layouts. In addition, the following specifications for elearning technologies might be considered:

- quality or design requirements for new applications,

- maximum time that a web page takes to load,

- maximum graphic size per web page.

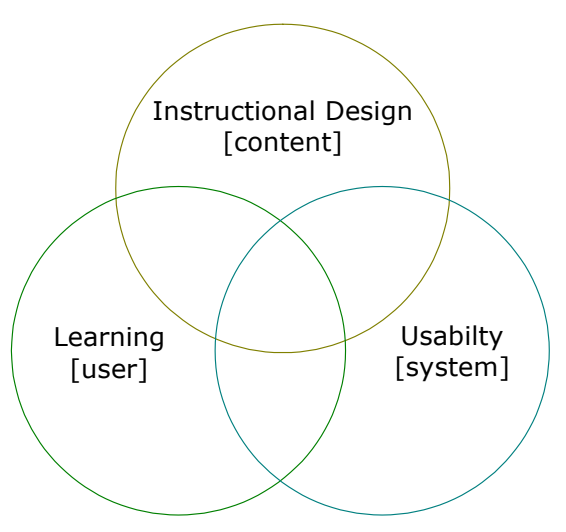

Figure 2. The e-learning instructional design process (adapted from [7])

In the next section, we will provide recommendations on to ensure consistency across individual tiers.

Arguably, the more difficult question relates to the module's instructional design. As Clark and Mayer [2] point out, the instructional design approach is dependent on the educational goals for the module. More specifically, they identify two categories of learning: (1) inform programs where the goal is to build awareness or provide information, and (2) perform programs where the goal is to build specific skills. Perform programs are further divided into procedural or near-transfer skills (e.g., computer skills training) and principle-based or far-transfer skills that do not have one correct approach or outcome [2].

Engineering design typically falls in the latter fartransfer category, especially in the case of tier 3 modules. However, both inform and perform goals are relevant to CDEN modules as illustrated in Figure 3.

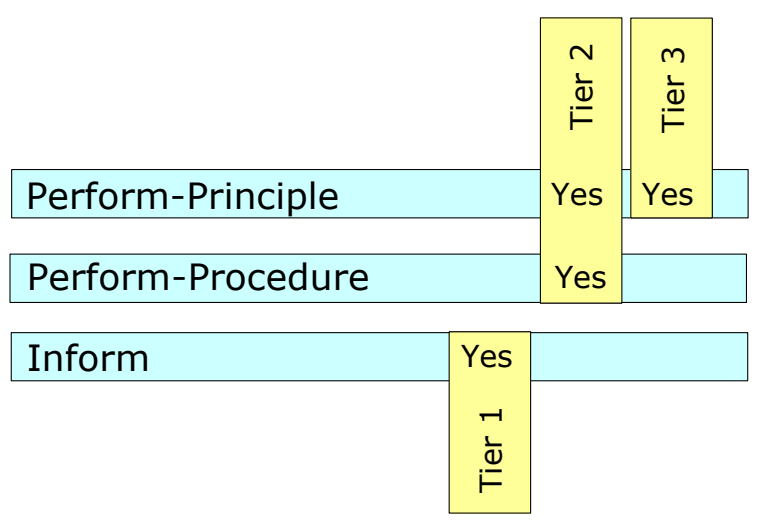

Figure 3. e-learning goals and the CDEN tiers 


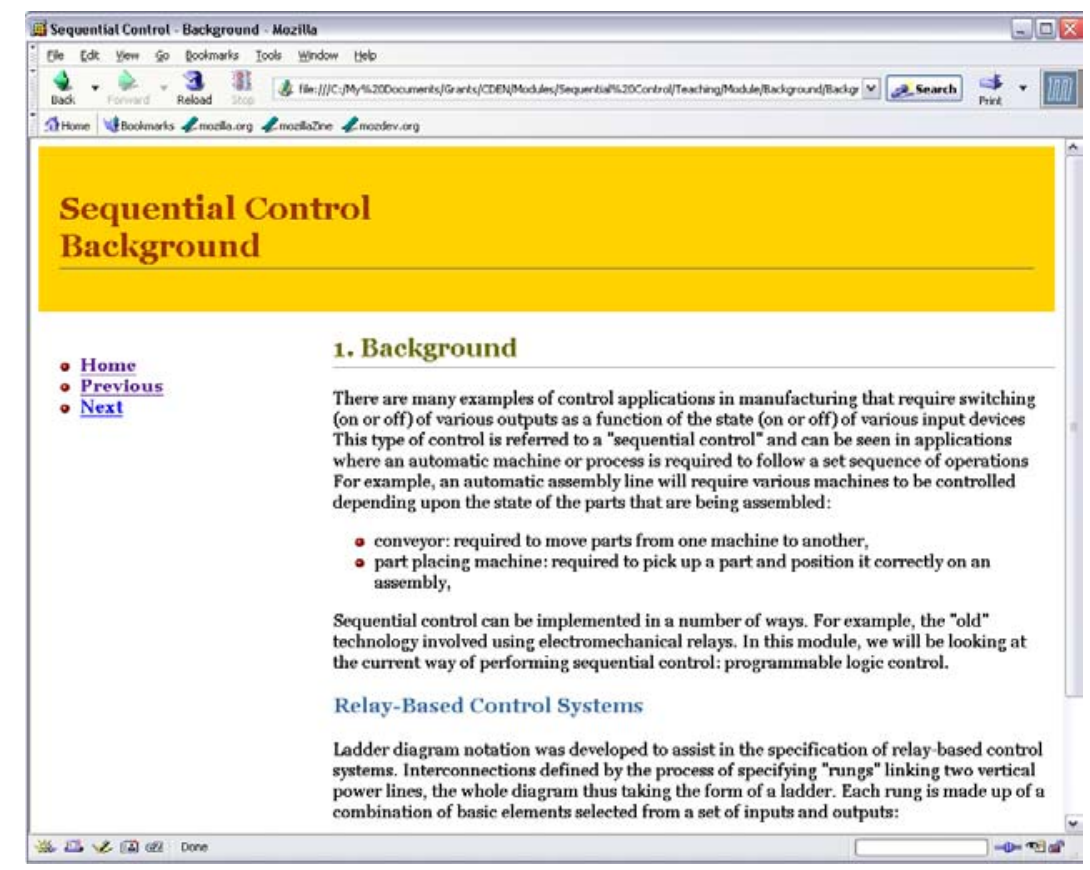

Figure 4. A tier 1 module with inform e-learning goals

In order to illustrate this approach to module delivery, we can look at an example of a flexible cutoff saw. In this case, the flexible cut-off saw can be thought of as a mechatronic system that integrates various subsystems: i.e., basic drive systems, position and velocity measurement, simple logic, state machines and programmable logic controllers (PLC's), basic manufacturing issues, and safety issues in machinery design. The cut-off saw system is intended to be flexible and modular in nature. For example, it should be possible to vary the size and complexity of the problem as well as the level and type of automation used (e.g., from zero to full axis 5 CNC). One might for instance consider just a straight cut to length arrangement, with or without automated sensing of stock, or one might wish to cope with compound angles on each end of the stock (and the 4 or 5 axis of control required). As well, the cut-off saw system should include some form of automated loading.

Figure 4 provides an example of an CDEN tier 1 module that is used to support the flexible cut-off saw project. In this example, the module provides the background on PLC (programmable logic controller) programming needed to understand the tier 2 and tier 3 modules associated with the flexible cut-off saw project. This tier 1 module would fall in the inform goals category as it provides a lot of information to the user, but limited practice opportunities [2].
The case study of the linear/rotational stage embodiment design shown in Figure 5 provides an example of a tier 2 module that uses a guided discovery approach to instruction. In this case, the student can explore the various sub-systems that make up the linear/rotational stage design. This module falls into the perform-principle goals category given its reliance on job-realistic problems and supporting resources [2]. As Clark and Mayer [2] note, with this type of instruction it is 'the instructor's job to serve as the cognitive guide and the learner's job to make sense of the presented material in the context of solving a job-related problem".

Tier 2 modules may also fall into the performprocedure goals category as illustrated in Figure 3. For example, the module tier 2 "position control" module shown in Figure 6 requires frequent responses from its users and provides immediate feedback (in this case, in the form of Quicktime video feeds).

As noted previously, a tier 3 module would integrate tier 1 and tier 2 design modules. For example, a tier 3 module for the flexible cut-off saw may be a description of the product architecture with links to various tier 1 and tier 2 modules. Alternatively, the tier 3 module may simply pose the design problem to the user, then help guide her through the design process (using tier 1 and tier 2 modules to support the user feedback). As a result, tier 


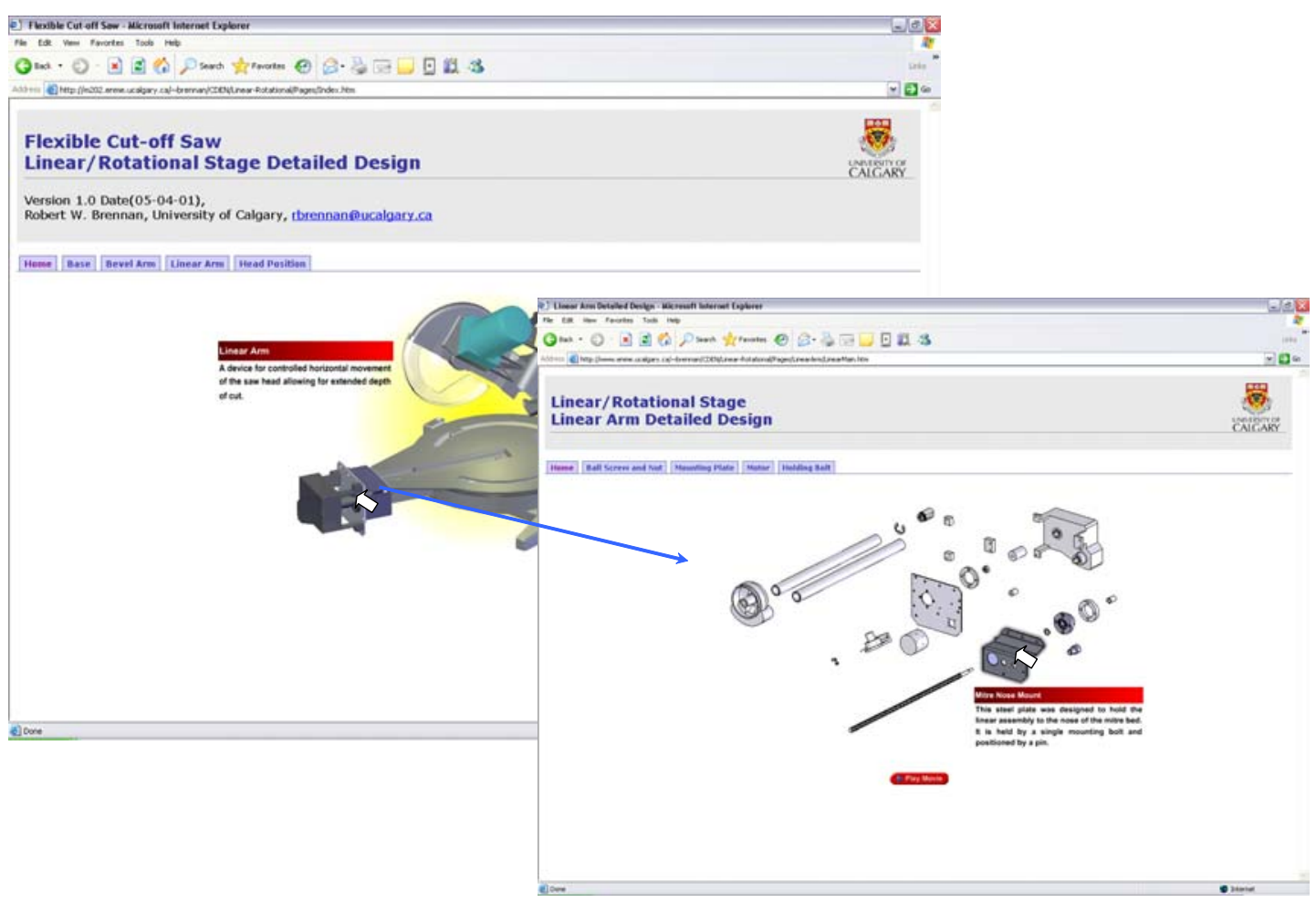

Figure 5. A tier 2 module with perform-principle e-learning goals

3 modules primarily focus on perform-principle goals as illustrated in Figure 3.

\section{Web Standard Solutions}

As noted previously, it is expected that CDEN module developers will have considerable expertise in the content area of their respective modules, however their expertise in the development of web-based design courseware may vary considerably. Despite this, their teaching material will need to be converted to a format that is accessible on the internet, is usable, and is preferably consistent with other CDEN modules.

To ensure consistent courseware, a rigid standard, based on a widely-understood format such as PDF (Portable Document Format) [8] could be used. This approach is similar to that used for ensuring consistent formatting of papers in conference proceedings or journals. The main drawback is that this approach does not fully take advantage of the technical capabilities of the internet and is arguably not that different from course notes or a textbook.

Alternatively, CDEN module developers could be allowed complete latitude in the formatting of their courseware provided that it can be rendered in a standard web browser. This approach would certainly provide module developers the flexibility to take advantage of a wide range of e-learning technology but would most certainly result in a very disparate set of modules. As well, many potential module authors may feel that the learning curve required to transform their content into comparable web-based courseware is too steep.

The first approach is similar to that used in the MIT Open Courseware Project [9]. For example, the majority of its courseware is presented in a similar fashion to typical course websites: i.e., course syllabi and PDF versions of lecture notes.

The CDEN project differs in its specific focus on engineering design and, as a result, its heavier weighting of principle-based or far-transfer skills. As well, design modules are typically highly interrelated and need to be accessed by users as such.

As a result, CDEN module developers typically require more flexibility that the first option can offer. In some cases such as modules with inform goals, a basic PDF, text-based module may be suitable, however a wider variety of options are certainly desirable in other cases. How can we ensure consistency and relatively seamless authoring though?

To address this question, we propose a courseware authoring approach that is based on web standards. By "web standards" we mean "common protocols that 


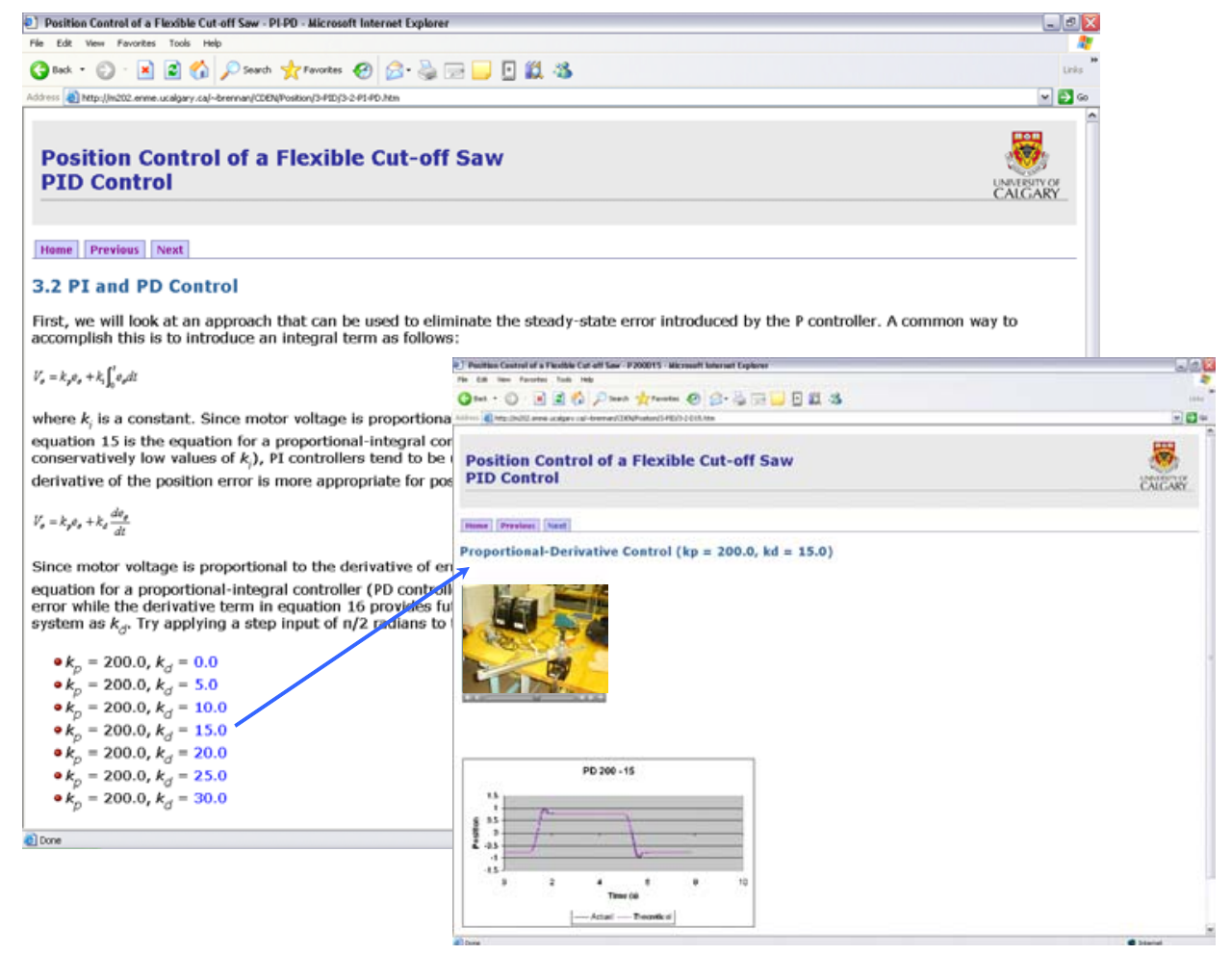

Figure 6. A tier 2 module with perform-procedure e-learning goals

promote [the World Wide Web's] evolution and ensure its operability" [10]. Just as standardization of parts enabled mass production, web standards can facilitate the development of courseware that is accessible, usable, and consistent. More specifically, web standards include the following technologies [10]:

- $\quad$ Structured languages (XHTML, XML)

- Presentation languages (CSS)

- Object models (DOM)

- Scripting languages (JavaScript)

- Additional Presentation Languages (MathML, SVG)

- As well as emerging standards such as those for television- and PDA-based browsers.

In this section, we focus on the use of XHTML (eXtensible HyperText Markup Language) and CSS (Cascading Style Sheets) to simplify the "usability" aspect of module development discussed in the previous section. With this approach, the following benefits are possible [11]:

- reduced markup,

- increased separation of content and presentation,
- improved accessibility, and

- forward compatibility.

One of the challenges of designing a learning module is to maintain a consistent style and layout that can be easily updated even after the module is published. Cascading Style Sheets (CSS) are designed to separate form from function in web page development. Style sheets store information that controls the formatting/style of a page (e.g., font size, color, and object placement) separately from the content of the page itself. This information can be used for one or more pages. For example, depending on the type of style sheet, the author can change the style of an entire site just by modifying the style sheet, making it easy to create a uniform, consistent look throughout the module. Another advantage of using a CSS template is that the plain content of the page can still be viewed if the browser does not recognize CSS.

This is illustrated in Figure 7. For example, if the browser is not standards compliant (e.g., an old version) or if an alternative media type is used (e.g., PDA, Braille, speech synthesizer, etc.), the content can still be rendered. With web standards compliant browsers, a consistent "look and feel" can be achieved using a single CSS style sheet for pages of the same 


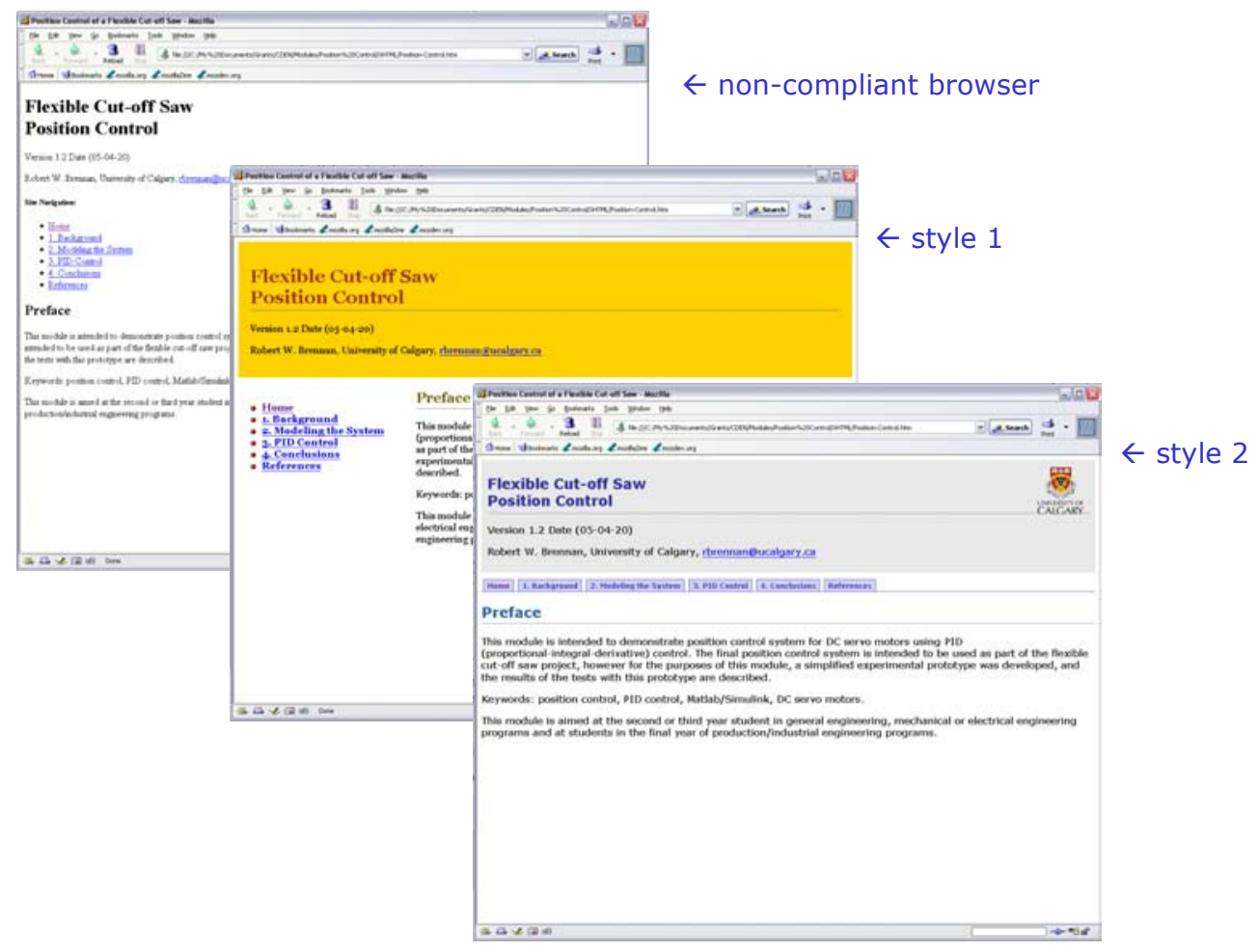

Figure 7. Various renderings of the same content with CSS

type. For example, in Figure 7, "style 1" may be used for tier 1 modules while "style 2 " may be used for tier 2 modules.

Another advantage of this approach is that it is easily accessible by potential module authors: i.e., it does not require a steep learning curve to author new modules. In contrast to standard HTML (HyperText Markup Language), CSS provides a simple framework that can be used by non professionals that does not require a specific web authoring application such as Macromedia's Dreamweaver [12] or Microsoft's FrontPage [13].

For the examples shown in Figrues 5-7 we used CSS files to define the font styles, custom bullets, background color scheme, logo image, link buttons, and page layout of the module. The CSS style sheets would be prepared in advance for each tier and made available on the CDEN e-Design portal [14], leaving only the content to the module's author. For example, the module author could use an XHTML template similar to the one shown on the left of Figure 8 and simply insert the content in the appropriate locations. Once the content is added, an appropriate CSS style sheet could then be selected for the module. As noted previously, the choice of CSS style sheet could be dependent upon the module's tier.

\section{Conclusions and Next Steps}

In this paper we have provided an overview of the current work that is being conducted by the Canadian Design Engineering Network (CDEN) to develop engineering design courseware or "CDEN modules" for use in Canadian engineering schools as well as our work on creating guidelines for the authors of these modules. Considerable design courseware has already been developed by members of CDEN, however to this date the usability of these modules is inconsistent. The three-tiered module classification system and the use of web standards for authoring described in this paper show promise as a solution to this problem.

Once more CDEN modules come online the issue of managing these modules will become a concern. For example, given a large number of seemingly unrelated modules, is it possible to establish a single point of entry (i.e., a server) that provides a sense of structure and context around the various topics and tiers? To address this, the author has been working closely with the University of Calgary's Learning Commons [15] on the development of an "e-Design Portal" for CDEN modules [14]. 


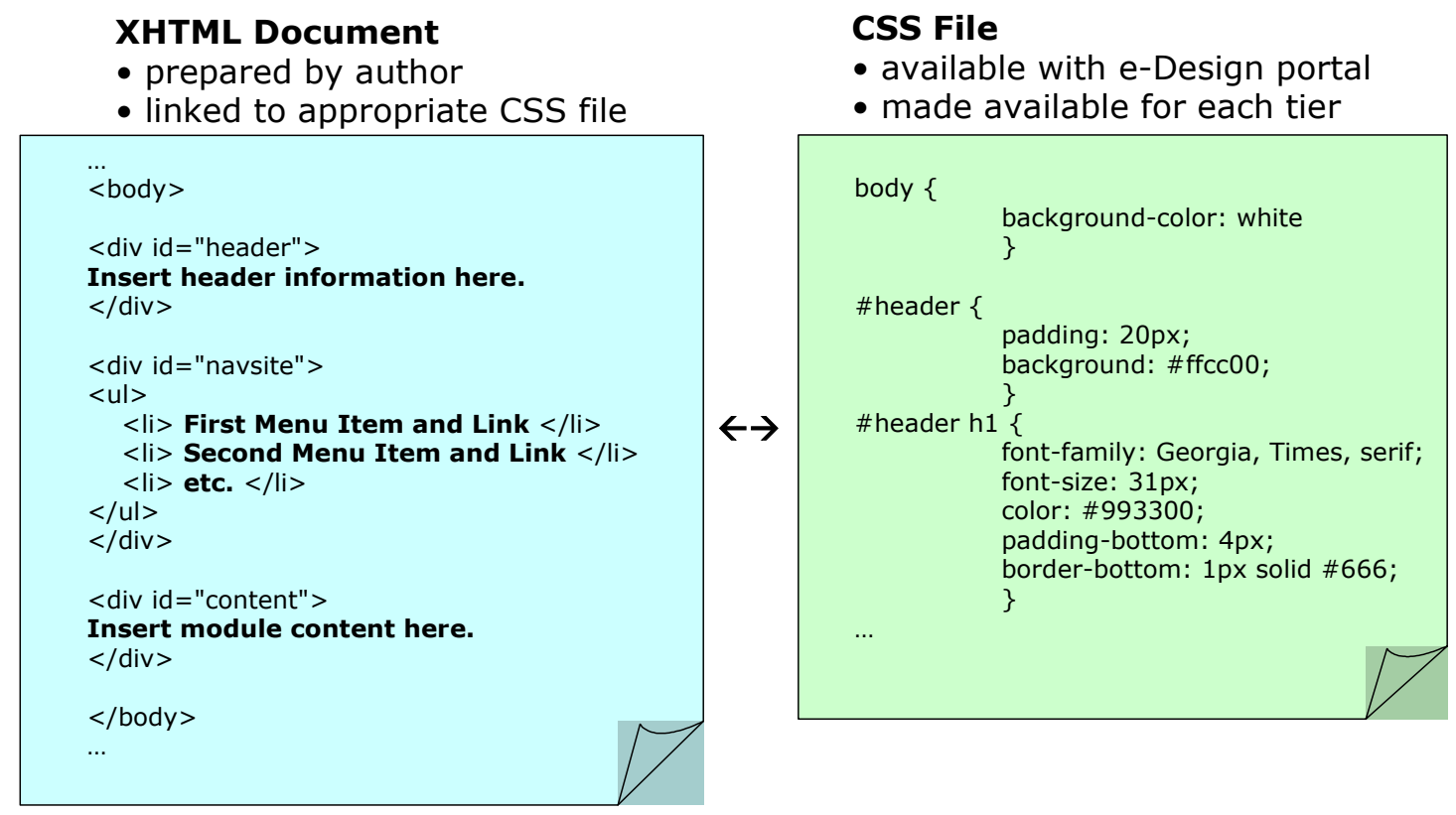

Figure 8. Authoring a CDEN module using web standards

The first draft of the CDEN e-Design Portal is scheduled to go online in early June 2005. At that time, existing CDEN Design Modules from CDEN members will be added to the database and made available for review. Modules formatted using $\mathrm{XHTML} / \mathrm{CSS}$ as describe in this paper will have the added advantage at this time of facilitating better indexing and searching of their content: i.e., search engines love heading tags and also index $<$ title $>$ and $<$ meta $>$ tags typical of XHTML markup.

Based on the review of the published CDEN modules, the module guidelines will be refined in order to provide a relatively consistent interface to users. This work will culminate in the development of a set of CDEN module development guidelines with associated CSS style sheets. As well, the impact of the e-Design Portal on engineering design education in Canada will then be investigated through CDEN partner universities across Canada.

\section{Acknowledgements}

The design work on aspects of this project was conducted by Wiebe Boomsma and Michiel Huizer of the Technische Universiteit Eindhoven, Department of Mechanical Engineering and Sean Barton and Steven
Sharpe of the University of Calgary, Department of Mechanical Engineering.

The authors wish to thank the Natural Sciences and Engineering Research Council of Canada and the University of Calgary, Faculty of Engineering for their generous support of this research.

\section{References}

[1] Yellowley, I., R.D. Venter, F.A. Salustri, "The Canadian design engineering network (CDEN/RCCI): sharing engineering design educational tools within thirty three schools of engineering in Canada", Proceedings of the 8th World Conference on Continuing Engineering Education, 2001.

[2] Clark, R.C. and R.E. Mayer, e-Learning and the Science of Instruction, Pfeiffer, 2003.

[3] Yellowley, I. and P. Gu, "Design directed engineering education", Proceedings of the Inaugural CDEN Design Conference, Montreal, July 29-30, 2004.

[4] Angeles, J., R. Britton, L. Chang, F. Charron, P. Gregson, P. Gu, P. Lawrence, W. Stiver, D. Strong, P. Stuart, B. Thompson, "The engineering design competency", Proceedings of the Inaugural CDEN Design Conference, Montreal, July 29-30, 2004.

[5] IMS Global Learning Consortium, IMS Meta-data Best Practice Guide for IEEE 1484.12.1-2002 Standard for Learning Object Metadata, Version 1.3 Public Draft, 
http://www.imsglobal.org/metadata/mdv1p3pd/imsmd bestv1p3pd.html, 2004.

[6] http://derekstockley.com/elearning-definition.html

[7] Koohang, A.and Du Plessis, Jacques. "Architecting Usability Properties in the e-Learning Instructional Design Process", International Journal on E-Learning, July-September 2004, pp. 38-44.

[8] http://www.adobe.com/

[9] http://ocw.mit.edu/

[10] http://www.webstandards.org/

[11] Cederholm, Web Standards Solutions: the Markup and Style Handbook, Friends of Ed, 2004.

[12] http:// www.macromedia.com/

[13] http:// www.microsoft.com/frontpage/

[14] Brennan, R.W., "An internet interface for engineering design courseware," Proceedings of the 25th ASME Computers and Information in Engineering Conference, September 24-28, 2005.

[15] http://commons.ucalgary.ca/ 